\title{
Adaptação de lente de contato gelatinosa especial para ceratocone
}

\author{
Keratoconus special soft contact lens fitting
}

\author{
Ester Sakae Yamazaki ${ }^{1}$ \\ Vanessa Cristina Batista da Silva ${ }^{2}$ \\ Vagner Morimitsu ${ }^{3}$ \\ MarceloSobrinho ${ }^{4}$ \\ Nelson Fukushima ${ }^{5}$ \\ César Lipener ${ }^{6}$
}

\section{RESUMO}

Objetivo: Relatar a experiência inicial com o uso de lentes de contato gelatinosas especiais para ceratocone. Métodos: Estudo retrospectivo de 80 olhos de 66 pacientes usuários de lentes de contato gelatinosas especial para ceratocone, no Setor de Lentes de Contato da UNIFESP e em clínicas particulares. Os pacientes foram classificados de acordo com o grau de gravidade da doença pela avaliação ceratométrica. Foram coletados os seguintes dados: idade, sexo, diagnóstico, ceratometria, acuidade visual com a lente, equivalente esférico (ES), curvatura da lente e indicação para o seu uso. Resultados: Foram estudados 80 olhos de 66 pacientes usuários de lente gelatinosa para ceratocone. A média de idade foi de 29 anos, sendo 51,5\% homens e 48,5\% de mulheres. De acordo com os grupos: incipiente: $15,0 \%$, moderado: $53,7 \%$, avançado: $26,3 \%$, grave: $5,0 \%$. A maioria dos olhos estudados $(91,25 \%)$ apresentou acuidade visual melhor que 20/40 com a lente. Foram adaptadas $58 \%$ lentes de poder esférico (média de $-5,45$ dioptrias) e $41 \%$ lentes com grau esferocilíndrico (variando de -0,50 a-5,00 dioptrias cilíndricas). A curva esférica mais utilizada foi de $7,6 \mathrm{~mm}$ (61\% dos olhos). As principais razões para a escolha e adaptação desta lente foram devido à intolerância e ao padrão de adaptação insatisfatório com outras lentes. Conclusão: A lente gelatinosa para ceratocone pode ser usada em casos de difícil adaptação, como uma opção para melhorar o conforto e possibilitar a reabilitação visual em pacientes que não obtiveram alternativas a não ser algum procedimento cirúrgico.

Trabalho realizado no Setor de Lente de contato Refração do Departamento de Oftalmologia da Universidade Federal de São Paulo - UNIFESP - São Paulo (SP) - Brasil.

${ }^{1}$ Estagiário do Setor de Lente de Contato e Refração do Departamento de Oftalmologia da Universidade Federal de São Paulo - UNIFESP - São Paulo (SP) - Brasil.

${ }^{2}$ Estagiário do Setor de Lente de Contato e Refração do Departamento de Oftalmologia da UNIFESP - São Paulo (SP) - Brasil.

${ }^{3}$ Estagiário do Setor de Lente de Contato e Refração do Departamento de Oftalmologia da UNIFESP - São Paulo (SP) - Brasil.

${ }^{4}$ Médico colaborador do Setor de Lente de Contato e Refração do Departamento de Oftalmologia da UNIFESP - São Paulo (SP) - Brasil.

5 Médico colaborador do Setor de Lente de Contato e Refração do Departamento de Oftalmologia da UNIFESP - São Paulo (SP) - Brasil.

${ }^{6}$ Mestre em Oftalmologia e Chefe do Setor de Lente de Contato e Refração do Departamento de Oftalmologia da UNIFESP - São Paulo (SP) - Brasil

Endereço para correspondência: Ester Sakae Yamazaki. R. Estero Belaco, 274/31 - São Paulo (SP) CEP 04145-020 E-mail: ester.yamazaki@gmail.com

Recebido para publicação em 26.09.2005

Versão revisada recebida em 12.12.2005

Aprovação em 20.02.2006

Os autores não têm nenhum interesse comercial nos produtos apresentados neste estudo.
Descritores: Lentes de contato hidrofílicas; Acuidade visual; Transtornos da visão, Doenças da córnea; Ceratocone/reabilitação; Acomodação ocular

\section{INTRODUÇÃO}

O ceratocone foi primeiramente descrito como uma doença produzindo afinamento corneano, por Nottingham $(1854)^{(1)}$. As primeiras lentes de contato utilizadas na correção de ceratocone foram descritas por Adolf Fick $(1888)^{(2)}$. Uma revisão sobre ceratocone em 1984, caracterizou a doença como progressiva, bilateral, assimétrica, não inflamatória, com aumento da curvatura corneana, afinamento apical e cicatrizes centrais na córnea ${ }^{(3)}$. Essas alterações freqüentemente levam à redução da visão devido ao astigmatismo irregular e cicatriz corneana central. O diagnóstico de ceratocone pode ser feito considerando a ceratometria maior que 47,20 dioptrias (D), encurvamento da córnea inferior maior que 1,20 D comparado com a córnea superior e mudança do eixo radial do astigmatismo maior que 21 graus $^{(4)}$.

O tratamento clínico do ceratocone depende da severidade da doença 
que pode ser classificada de acordo com os valores ceratométricos em incipiente, moderado, avançado e grave ${ }^{(5)}$. Em boa parte dos casos de ceratocone incipientes a avançados, a acuidade visual pode ser corrigida tanto com os óculos quanto com o uso de lentes de contato. As lentes de contato que tem sido mais utilizadas são as lentes rígidas gás permeáveis (LCRGP) por possibilitar melhora da correção visual ${ }^{(6)}$. Porém, existem casos em que a adaptação de lentes é mais difícil, principalmente em pacientes intolerantes ao uso de lentes rígidas, e/ou padrão de adaptação ruim, o que pode impossibilitar o uso das lentes. Considerando que grande parte da população portadora de ceratocone é composta por indivíduos jovens em fase economicamente ativa, torna-se uma tarefa muito importante a reabilitação visual nesses casos. Nos estágios avançados da doença, a visão pode ser incompatível com o trabalho ou com os estudos, segundo alguns autores ${ }^{(3-7)}$.

O objetivo deste estudo é avaliar a adaptação de uma lente gelatinosa especial para ceratocone em casos que não tiveram sucesso com outras lentes, em busca do maior conforto, melhor padrão de adaptação e qualidade de visão.

\section{MÉTODOS}

Foi realizado um estudo retrospectivo em 80 olhos (66 pacientes), usuários de lente de contato Perfect Keratoconus (PK) / World Vision, no Setor de Lentes de Contato da Universidade Federal de São Paulo/Escola Paulista de Medicina e em clínicas particulares, no período de janeiro 2004 a junho 2005. Foram coletados os seguintes dados: idade, sexo, diagnóstico, ceratometria, acuidade visual com as lentes, equivalente esférico (ES), curvatura da lente e indicação para o seu uso. Os pacientes portadores de ceratocone foram classificados de acordo com a ceratometria $(\mathrm{KT})^{(5)} \mathrm{em}$ : incipiente $(\mathrm{KT}<45,00$ dioptrias (D) nos dois meridianos), moderado (KT entre 45,00 e 52,00 D nos dois meridianos), avançado (KT entre 52,00 e 62,00 D nos dois meridianos) e grave (KT>62,00 D nos dois meridianos). Quando os dois meridianos não se encontraram dentro do mesmo grupo, classificou-se pelo meridiano mais curvo. Os parâmetros e propriedades da lente estão demonstrados no quadro 1 .

A adaptação da lente foi realizada segundo critérios do fabricante: (aguardando uma hora para exame) observando o movimento da lente entre $0,70-1 \mathrm{~mm}$ ao piscar, sua centralização, ausência de bolha sob a lente e bordas não elevadas.

\section{RESULTADOS}

Dentre os 80 olhos estudados (66 pacientes), 34 (51,5\%) eram do sexo masculino e $32(48,5 \%)$ do sexo feminino. A idade variou entre 14 a 51 anos, sendo a média de 29 anos, o que é compatível com o início desta doença. Com relação ao diagnóstico, todos eram portadores de ceratocone, sendo 6 olhos (9\%) de pacientes portadores de ceratocone submetidos a implante de anel intra-estromal. Os pacientes com anel foram classificados de acordo com os valores ceratométricos pósoperatórios da mesma forma que os demais pacientes do trabalho. Desse total de 6 olhos com anel, 1 olho pertencia ao grupo incipiente, 2 olhos ao grupo moderado e 3 olhos ao grupo avançado (Tabela 1).

Quanto aos grupos: ceratocone incipiente 12 olhos $(15,0 \%)$, moderado 43 olhos $(53,75 \%)$, avançado 21 olhos $(26,3 \%)$ e grave 4 olhos $(5,0 \%)$, distribuídos no gráfico 1 .

Em relação aos resultados visuais (Tabela 2); dos 80 olhos estudados, 36 olhos (45\%) alcançaram acuidade visual melhor ou igual a 20/25, 73 olhos $(91,25 \%)$ atingiram visão melhor que 20/40 com a lente. Já os 7 olhos restantes $(8,75 \%)$ apresentaram visão entre $20 / 50$ e 20/80. Pacientes que apresentaram acuidade visual com a lente melhor que 20/30 por grupo: incipiente

\begin{tabular}{|c|c|}
\hline \multicolumn{2}{|c|}{$\begin{array}{l}\text { Quadro 1. Os parâmetros e propriedades da lente Perfect Keratoco- } \\
\text { nus }^{\mathrm{TM}} \text { conforme descrito pelo fabricante }\end{array}$} \\
\hline \multicolumn{2}{|l|}{ Propriedades } \\
\hline Material & $\begin{array}{l}\text { Xylofilcon a } 67 \% \mathrm{H}_{2} \mathrm{O} \\
\text { Glicerol não-iônico GM3 } \\
\text { (GMMA+VP) } 58 \% \mathrm{H}_{2} \mathrm{O}\end{array}$ \\
\hline Permeabilidade ao oxigênio & DK escala fatt $21,5^{-11}$ a $35^{\circ} \mathrm{C}$ \\
\hline \multicolumn{2}{|l|}{ Parâmetros } \\
\hline Curva esférica & CB 7,30 / 7,60 / 7,90 / 8,20 \\
\hline Diâmetro & $14,20 \mathrm{~mm}$ \\
\hline Dioptrias esféricas & $+10,00$ a $-20,00$ esf \\
\hline Zona óptica & $8,30 \mathrm{~mm}$ \\
\hline Espessura central $(-3,00)$ & $0,36 \mathrm{~mm}$ \\
\hline Espessura de borda & $0,11 \mathrm{~mm}$ \\
\hline Curva tórica & $\begin{array}{l}\text { cil }-0,50 \text { a }-2,50 \text { eixos } \\
\text { de } 1 \text { a } 180 \text { passos de } 1 \text { grau }\end{array}$ \\
\hline \multicolumn{2}{|l|}{ Desenho da lente } \\
\hline & $\begin{array}{l}\text { curva base: bicurva, } \\
\text { zona óptica: esférica, } \\
\text { zona periférica: asférica }\end{array}$ \\
\hline
\end{tabular}

\begin{tabular}{|lccccc|}
\hline \multicolumn{4}{c|}{ Tabela 1. Pacientes portadores de anel em uso da lente gelatinosa para cone } \\
Casos & Grau do cone* & Idade & Sexo & Curva base / Poder da lente & AV \\
1 & Incipiente & 30 & $\mathrm{~F}$ & $7,9 /-5,00 \mathrm{DE}$ & $20 / 40$ \\
2 & Moderado & 27 & $\mathrm{~F}$ & $7,6 /-4,50 \mathrm{DE}-1,50 \mathrm{DC} \times 40$ \\
3 & Moderado & 23 & $\mathrm{M}$ & $7,9 / 0,00 \mathrm{DE}-2,00 \mathrm{DC} \times 55$ \\
4 & Avançado & 30 & $\mathrm{~F}$ & $7,9 /-6,75 \mathrm{DE}-3,00 \mathrm{DC} \times 100$ \\
5 & Avançado & 44 & $\mathrm{~F}$ & $7,6 /-19,00 \mathrm{DE}$ & $20 / 25$ \\
6 & Avançado & 38 & $\mathrm{M}$ & $7,3 /-4,75 \mathrm{DE}$ & $20 / 40$ \\
*baseado em valores keratométricos pós-operatórios & & & & \\
\hline
\end{tabular}


$8(66,6 \%)$ de 12 olhos, moderado $38(88,4 \%)$ de 43 olhos, avançado $15(71,4 \%)$ de 21 olhos, grave apenas 1 (25\%) de 4 olhos.

Quanto ao poder dióptrico das lentes, do total de 80 olhos adaptados com lente especial para ceratocone, em 47 olhos $(58,75 \%)$ foi usada lente com grau esférico (média de $-5,45$ dioptrias esféricas) e, em 33 olhos $(41,25 \%)$, foi usada lente com grau esferocilíndrico que variou de $-0,50$ a -5,00 dioptrias cilíndricas.

Em relação à curva esférica foram adaptadas 16 lentes (20\%) com curva 7,3, 49 lentes $(61,25 \%)$ com curva 7,6 e 15 lentes $(18,75 \%)$ com curva 7,9 (Tabela 3$)$.

A principal indicação das lentes gel foi devido à intolerância do paciente às lentes rígidas, acompanhada de queixas de desconforto. Outra razão para a escolha dessas lentes foi o insucesso no padrão de adaptação com outras lentes, sejam elas rígidas, gelatinosas, híbridas ou adaptação à cavaleiro (piggyback).

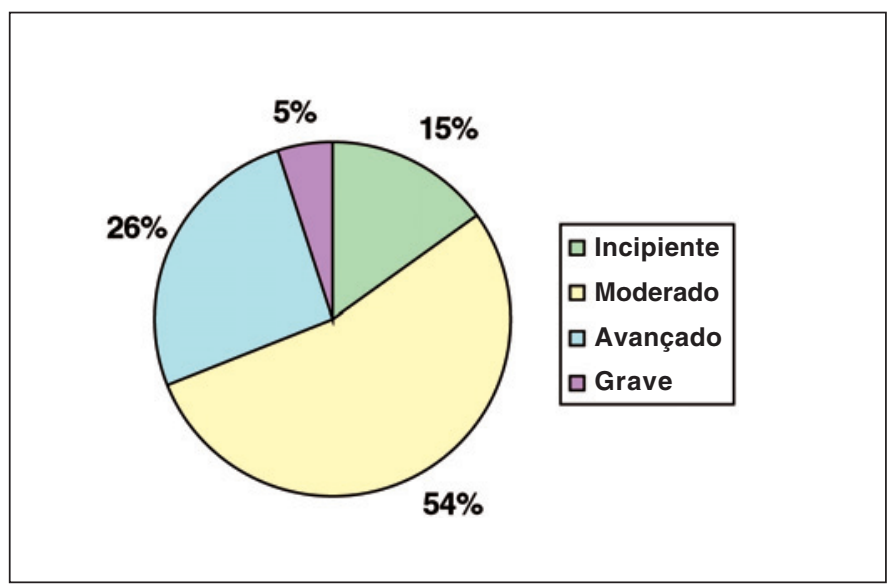

Gráfico 1 - Classificação dos cones quanto à ceratometria

\section{DISCUSSÃO}

Este estudo mostra a reabilitação visual em pacientes usuários de lentes gelatinosas especiais para ceratocone, possibilitando mais uma alternativa no tratamento clínico de pacientes com ceratocone. Foi também possível a adaptação de lentes em pacientes com implante de segmento de anel intra-estromal ${ }^{(8)}$.

Um estudo sobre a adaptação em cone, utilizou lentes rígidas monocurvas em 529 olhos $(60,04 \%)$ seguidas pelas bicurvas em 113 olhos (12,83\%). Nos casos de insucesso com as lentes rígidas e gelatinosas, utilizou-se o sistema à cavaleiro (piggyback) e raramente a lente híbrida (tipo softperm) ${ }^{(9)}$. Em relação ao sistema à cavaleiro (piggyback: lente rígida gáspermeável de alto DK sobre lente gelatinosa), suas funções são aliviar o desconforto, melhorar o posicionamento e auxiliar a estabilidade da lente rígida, além de proteger a córnea em caso de erosões recorrentes, freqüentes nos ceratocones centrais em

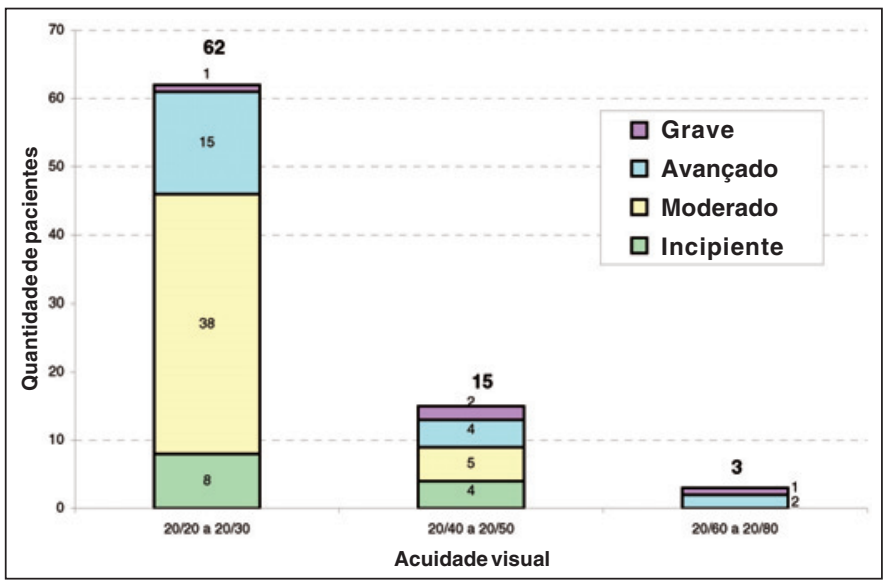

Gráfico 2 - Acuidade visual atingida por grupos de pacientes

\begin{tabular}{|c|c|c|c|c|c|}
\hline & Incipiente & Moderado & Avançado & Grave & Total de olhos \\
\hline $20 / 20$ & $3(3,75 \%)$ & $4(5,00 \%)$ & $2(2,50 \%)$ & $0(0,00 \%)$ & $9(11,25 \%)$ \\
\hline $20 / 25$ & $2(2,50 \%)$ & $16(20,00 \%)$ & $9(11,25 \%)$ & $0(0,00 \%)$ & $27(33,75 \%)$ \\
\hline $20 / 30$ & $3(3,75 \%)$ & $18(22,50 \%)$ & $4(5,00 \%)$ & $1(1,25 \%)$ & $26(32,50 \%)$ \\
\hline $20 / 40$ & $3(3,75 \%)$ & $5(6,25 \%)$ & $2(2,50 \%)$ & $1(1,25 \%)$ & $11(13,75 \%)$ \\
\hline $20 / 50$ & $1(1,25 \%)$ & $0(0,00 \%)$ & $2(2,50 \%)$ & $1(1,25 \%)$ & $4(5,00 \%)$ \\
\hline $20 / 60$ & $0(0,00 \%)$ & $0(0,00 \%)$ & $1(1,25 \%)$ & $1(1,25 \%)$ & $2(2,50 \%)$ \\
\hline $20 / 80$ & $0(0,00 \%)$ & $0(0,00 \%)$ & $1(1,25 \%)$ & $0(0,00 \%)$ & $1(1,25 \%)$ \\
\hline Total de olhos & $12(15,00 \%)$ & $43(53,75 \%)$ & $21(26,25 \%)$ & $4(5,00 \%)$ & $80(100,00 \%)$ \\
\hline
\end{tabular}

\begin{tabular}{|lcccrc|}
\hline & & Tabela 3. Relação curvas esféricas adaptadas nos grupos & \\
& Incipiente & Moderado & Avançado & Grave & Total de olhos \\
7,3 & $0((0,00 \%)$ & $7(8,75 \%)$ & $7(8,75 \%)$ & $2(2,50 \%)$ & $16(20,00 \%)$ \\
7,6 & $6(7,50 \%)$ & $28(35,00 \%)$ & $13(16,25 \%)$ & $2(2,50 \%)$ & $49(61,25 \%)$ \\
7,9 & $6(7,50 \%)$ & $8(10,00 \%)$ & $1(1,25 \%)$ & $0(0,00 \%)$ & $15(18,75 \%)$ \\
Total de olhos & $12(15,00 \%)$ & $43(53,75 \%)$ & $21(26,25 \%)$ & $4(5,00 \%)$ & $80(100,00 \%)$ \\
\hline
\end{tabular}


forma de bico (nipple cone). Em 90\% dos pacientes com cone avançado e grave que eram intolerantes às $\mathrm{LC}$ rígidas foram possíveis evitar cirurgia com o uso dessas lentes ${ }^{(10)}$.

As lentes de contato gelatinosas podem propiciar visão aceitável e conforto para os pacientes com ceratocone, provando ser um auxílio visual útil. A acuidade visual é mais satisfatória em cones de grande diâmetro do que em cones centrais de pequeno diâmetro. As lentes gelatinosas tóricas ficam mais bem adaptadas nos cones centrais, possivelmente por ter a córnea, com esse tipo de cone, uma configuração mais semelhante ao desenho padrão da lente de contato, facilitando o posicionamento e estabilidade do eixo. Deve-se lembrar, no entanto, que os resultados visuais no ceratocone com a adaptação das lentes gelatinosas comuns são inferiores aos das lentes rígidas ${ }^{(11)}$.

Apenas $10 \%$ a $20 \%$ dos pacientes com ceratocone necessitam de transplante de córnea, e na maioria deles a cirurgia pode ser postergada ou evitada pela adaptação de lentes de contato $^{(12-13)}$.

Uma das hipóteses para o relativo sucesso na presente amostra, pode ser o desenho desta lente, que proporcionou boa estabilização, apresentando dois pontos de fenestração para equilibrar a pressão entre a curva frontal e anterior da lente melhorando a liberação da lágrima e eliminando as bolhas de ar. Além disso, apresenta uma zona central com espessura elevada resultando em possível melhora da qualidade de visão. Porém a desvantagem seria a redução da transmissibilidade do oxigênio e complicações relacionadas à hipóxia corneana. Além do desenho bicurvo que poderia acomodar melhor os cones mais centrais, a borda e o material da lente propiciam um relativo conforto ao paciente, apresenta também uma zona intermediária com formato lenticular e anel em sua estrutura, centralizando a lente.

Devido ao escasso material publicado na literatura mundial sobre esta lente e pouco tempo de experiência no meio oftalmológico, é necessário maior tempo de seguimento para avaliar resultados em longo prazo e as eventuais complicações relacionadas ao uso crônico desta lente.

\section{CONCLUSÃO}

A utilização de lente de contato gelatinosa especial para ceratocone é mais uma opção para melhora da qualidade visual, do conforto e reabilitação visual em pacientes portadores de ceratocone, inclusive aqueles submetidos à implante de anel intra-estromal.

\section{ABSTRACT}

Purpose: To evaluate the fitting and use of a soft contact lens in keratoconic patients. Methods: Retrospective study on 80 eyes of 66 patients, fitted with a special soft contact lens for keratoconus, at the Contact Lens Section of UNIFESP and private clinics. Keratoconus was classified according to degrees of disease severity by keratometric pattern. Age, gender, diagnosis, keratometry, visual acuity, spherical equivalent (SE), base curve and clinical indication were recorded. Results: Of 66 patients ( 80 eyes) with keratoconus the mean age was 29 years, $51.5 \%$ were men and $48.5 \%$ women. According to the groups: $15.0 \%$ were incipient, $53.7 \%$ moderate, $26.3 \%$ advanced and $5.0 \%$ were severe. The majority of the eyes of patients using contact lenses $(91.25 \%)$ achieved visual acuity better than $20 / 40$. To 88 eyes $58 \%$ were tihed with lens with spherical power (mean -5.45 diopters) and $41 \%$ with spherocylinder power (from -0.5 to -5.00 cylindrical diopters). The most frequent base curve was 7.6 in $61 \%$ of the eyes. The main reasons for this special lens fitting were due to reduced tolerance and poor fitting pattern achieved with other lenses. Conclusion: The special soft contact lens is useful in fitting difficult keratoconic patients by offering comfort and improving visual rehabilitation that may allow more patients to postpone the need for corneal transplant.

Keywords: Contact lenses, hydrophilic; Visual acuity; Vision disorders; Corneal diseases; Keratoconus/rehabilitation; Accommodation, ocular

\section{REFERÊNCIAS}

1. Nottingham, J. Practical observations on conical cornea. London: Churchill; 1854. p.1-19.

2. Fick AE. A contact-lens. 1888 (translation) Arch Ophthalmol. 1988;106(10): 1373-7.

3. Krachmer JH, Feder RS, Belin MW. Keratoconus and related noninflammatory corneal thinning disorders. Surv Ophthalmol. 1984;28(4):293-322.

4. Rabinowitz YS. Keratoconus. Surv Ophthalmol. 1998;42(4):297-319.

5. Buxton JN, Keatles RH, Hoefle FB. The contact lens correction of keratoconus. In: Dabezies $\mathrm{OH}$ Jr. The Clao guide to basic science and clinical practice. Orlando: Grune \& Statton; 1984. p.55.1-14.

6. Leça RG, Fukushima N, Gonzaga R, Lipener C, Zamboni F, Lewinsky R, et al. Estudo clínico da adaptaçäo de lentes de contato no ceratocone. Arq Bras Oftalmol. 1995;58(3):149-51.

7. Zadnik K, Barr JT, Edrington TB, Everett DF, Jameson M, McMahon TT, et al. Baseline findings in the Collaborative Longitudinal Evaluation of Keratoconus (CLEK) Study. Invest Ophthalmol Vis Sci. 1998;39(13):2537-46.

8. Miranda D, Sartori M, Francesconi C, Allemann N, Ferrara P, Campos M. Ferrara intrastromal corneal ring segments for severe keratoconus. J Refract Surg. 2003;19(6):645-53.

9. Ghanem VC, Ghanem CC, Ghanem RC, Larinho C. Ceratocone: correlação entre grau evolutivo e padrão topográfico com o tipo de lente de contato adaptada. Arq Bras Oftalmol. 2003;66(2):129-35.

10. Tsubota K, Mashima Y, Murata H, Yamada M. A piggyback contact lens for the correction of irregular astigmatism in keratoconus. Ophthalmology. 1994; 101(1):134-9.

11. Koliopoulos J, Tragakis M. Visual correction of keratoconus with soft contact lenses. Ann Ophthalmol. 1981;13(7):835-7.

12. Kennedy RH, Bourne WM, Dyer JA. A 48-year clinical and epidemiologic study of keratoconus. Am J Ophthalmol. 1986;101(3):267-73.

13. Smiddy WE, Hamburg TR, Kracher GP, Stark WJ. Keratoconus. Contact lens or keratoplasty? Ophthalmology. 1988;95(4):487-92. 